\title{
Desarrollo Embriofetal del Iris: Inmunotinción del Morfógeno Shh
}

\author{
Embryo-fetal Development of Iris: Immunohistochemestry of Morphogen Shh
}

\author{
Mario Pellón ${ }^{1}$, Daniel Conei ${ }^{2,3,4}$; Gustavo Saint-Pierre² ${ }^{2}$ Carolina Smok $^{2,5}$; \\ Viviana Donoso²; Mariano del $\mathrm{Sol}^{3}$ \& Mariana Rojas ${ }^{2}$
}

PELLÓN, M.; CONEI, D.; SAINT-PIERRE, G.; SMOK, C.; DONOSO, V.; DEL SOL, M. \& ROJAS, M. Desarrollo Embriofetal del Iris: Inmunotinción del Morfógeno Shh. Int. J. Morphol., 37(1):221-226, 2019.

RESUMEN: Para que se desarrolle el iris, se requiere una especificación de la capa periférica de la copa óptica a un destino no neuronal y además la migración de células mesenquimales perioculares. Nuestro objetivo fue reconocer los cambios histológicos de los derivados periféricos de la copa óptica y mesénquima periocular, como también reconocer la presencia del morfógeno Sonic hedgehog (Shh) en las capas que constituyen el esbozo de iris. Se utilizaron 15 ratones hembras (Mus musculus) adultas jóvenes gestantes. Se realizó eutanasia con tiopental sódico. Los embriones y fetos de 12, 14,5 y 17 días post-coital (dpc) fueron procesados con técnica histológica e inmunohistoquímica con anticuerpo anti-Shh (scbt, H-160, conejo) con dilución 1:100 en PBS. A los 12 dpc, se observa una cópa óptica que presenta capas retinianas interna y externa, y el iris no se observa. Entre el cristalino y el ectodermo superficial se identifican 4 capas de células mesenquimales. A los 14,5 dpc, el iris contiene dos capas epiteliales (interna y externa) que se continúan con las capas neural y pigmentaria de la retina. Se observan 8 capas de células mesenquimales. A los 17 dpc, la capa epitelial interna del iris presenta un segmento más elongado con inmunotinción positiva a Shh y otra parte que constituye un epitelio de células cilíndricas simples negativas a este anticuerpo. La capa epitelial externa presenta el mismo epitelio inmunonegativo. Las capas de la retina también son positivas, como también la periferia del cristalino. No esta formado el iris ni tampoco el cuerpo ciliar. La inmunopositividad en el cristalino, en el primer segmento de la capa interna del esbozo del iris y en la capa ganglionar retinal a los $17 \mathrm{dpc}$, se relaciona con la diferenciación tardía del iris y con los ojos cerrados de las crías al nacimiento.

PALABRAS CLAVE: Iris; Bulbo ocular; Sonic hedgehog;Desarrollo Embrionario.

\section{INTRODUCCIÓN}

El iris tiene varias funciones, tales como regular la cantidad de luz que ingresa a las vías visuales y que llega a la retina, participar en el ajuste focal de los objetos más cercanos y estar implicado en la circulación del humor acuoso y en la regulación de la presión intraocular. Durante la embriogénesis, el iris se forma a partir de la copa óptica y del mesénquima periocular. A lo largo de este desarrollo, ocurre una especificación de la capa periférica de la copa óptica a un destino no neuronal, migración de células del mesénquima periocular circundante y la formación de músculos lisos del neuroectodermo (Cvekl \& Tamm, 2004; Davis-Silberman \& Ashery-Padan, 2008).
Durante el desarrollo del segmento anterior del bulbo ocular, las células que se originan desde el epitelio superficial necesitan interactuar con las células mesenquimales, que se originan principalmente en la cresta neural. Los defectos de la interacción epitelio-mesenquimática dan como resultado trastornos del desarrollo tales como la aniridia. La hipoplasia o ausencia del iris puede ser inducida experimentalmente en el ratón por deficiencia de la vitamina A materna (Cvekl \& Tamm).

Entre los factores de transcripción que se han identificado para participar en la coordinación del desarrollo anterior del bulbo ocular está PAX6, que es activo tanto en

\footnotetext{
${ }^{1}$ Departamento de Morfología, Facultad de Medicina, Universidad de La Frontera, Temuco, Chile.

${ }^{2}$ Laboratorio de Embriología Comparada, Programa de Anatomía y Biología del Desarrollo, Instituto de Ciencias Biomédicas, Facultad de Medicina, Universidad de Chile.

${ }^{3}$ Programa de Doctorado en Ciencias Morfológicas, Universidad de La Frontera, Temuco, Chile.

${ }^{4}$ Departamento de Ciencias Morfológicas, Facultad de Medicina y Ciencia, Universidad San Sebastián, Puerto Montt, Chile.

${ }^{5}$ Instituto de Investigación e Innovación en Salud, Facultad de Ciencias de la Salud, Universidad Central, Santiago, Chile.
} 
células epiteliales como mesenquimales durante el desarrollo ocular, aunque a diferentes dosis y etapas del desarrollo (Glaser et al., 1994). Por otra parte, la proteína morfogenética de hueso 4 (BMP4) y el factor de crecimiento transformante ß2 (TGF-ß2) están directamente involucrados en los procesos que controlan la morfogénesis en la parte anterior del bulbo ocular. BMP4 se expresa en iris, cuerpo ciliar y epitelio pigmentario de la retina de embrión y adulto (AsheryPadan et al., 2000), en cambio en TGF-B2 en fotorreceptores, microglia y células musculares lisas ubicadas en vasos sanguíneos (Sukhikh et al., 2010).

Sonic hedgehog (Shh) es un morfógeno, es decir, una molécula de naturaleza orgánica que es producida y secretada por un grupo de células embrionarias y que puede difundir y actuar a distancia sobre otras células o tejidos (Tabata \& Takei, 2004). En el bulbo ocular puede separar campos visuales a fines de tercera semana en la especie humana (Wallace, 2008). Variaciones en la señalización de Shh puede producir diversas malformaciones congénitas como holoprosencefalia, anomalías craneofaciales y ciclopia (Rimkus et al., 2016). Existen pocos estudios sobre el rol de Shh en el desarrollo ocular en etapas avanzadas. Nuestro objetivo fue reconocer los cambios histológicos graduales de los derivados periféricos de la copa óptica y del mesénquima periocular como también reconocer la presencia del morfógeno Sonic hedgehog (Shh) en las capas que constituyen el esbozo de iris en los períodos más avanzados del desarrollo.

\section{MATERIAL Y MÉTODO}

Se utilizaron 15 ratones hembra (Mus musculus) adultas jóvenes. Estas se colocaron en jaulas con machos reproductivamente sanos y jóvenes. Se consideró como el día 1 post-coital el momento en que se verifica la presencia de tapón mucoso a nivel vaginal, lo que significa que hubo cópula. A los 12, 14,5 y 17 días post-coital (dpc), los ratones gestantes se anestesiaron y se practicó eutanasia con tiopental sódico. Los embriones y fetos fueron fijados en formalina al $10 \%$ tamponada en PBS e incluidos en paraplast. Se obtuvieron cortes de $5 \mu \mathrm{m}$ con micrótomo Microm (HM315R), los cuales se adhirieron a portaobjetos xilanizados y con carga positiva (Citoglas). Fueron adheridos 4 cortes por portaobjeto, considerando un total de 3 portaobjetos por muestra. Se realizó tinción histológica con HematoxilinaEosina y técnica inmunohistoquímica. La recuperación antigénica fue realizada en vaporera por 40 minutos, con los cortes sumergidos en solución desenmascarante de antígenos (Vector Labs, H-3301). Se realizó bloqueo de peroxidasa endógena con peróxido de hidrógeno en metanol y bloqueo inespecífico de proteínas con PBS+BSA al $3 \%$. Para la incubación con el anticuerpo primario SHH (Santa Cruz Biotechnology, H-160, conejo) se usó una dilución 1:100 en PBS. Para la detección del anticuerpo primario se incubó con polímero conjugado con anticuerpo anti-conejo y HRP durante 15 minutos (SuperPictureTM, Thermo Fisher, 878963). Como sustrato para la HRP se usó diaminobencidina (DAB, Vector Labs, SK-4100). El control negativo estuvo dado por el desarrollo de la técnica completa, pero sin considerar el anticuerpo primario. Como control positivo se consideró la notocorda. Los cortes transversales seriados fueron analizados si resultaron marcados positivamente y fueron descritos morfológicamente

Los cortes histológicos fueron fotografiados en un microscopio óptico Leica ICC50 HD conectado a un computador. Se realizó una descripción morfológica de carácter cualitativo de las células del iris y se midió la altura de la capa interna del iris, capa externa del iris, capa nerviosa y capa pigmentaria de la retina con software ImageJ $1.50 \mathrm{i}$ (NIH, EE.UU.), presentándose como media y desviación estándar. Se utilizó test de ANOVA de dos vías y post-test de Tukey, con el software GraphPad Prism 6 (GraphPad Software Inc., San Diego, CA, EE.UU), considerándose un valor de $\mathrm{p}<0,05$ como significativo.

\section{RESULTADOS}

A los 12 dpc se observa la cópa óptica con sus dos hojas capa retiniana interna $(\mathrm{CRI})$, capa retiniana externa (CRE) y lente. Entre la retina y el lente se observa una matriz extracelular rica en glicosaminoglicanos, en cuyo espesor se observan vasos sanguíneos con glóbulos rojos nucleados. Entre el lente y el ectodermo superficial se identifican 4 capas de células mesenquimales (Fig. 1). La altura de la CRI en su porción periférica fue de 36,069 $\pm 1,936$ $\mu \mathrm{m}$ y en la porción central $67,326 \pm 3,264 \mu \mathrm{m}$, y la CRE periférica $13,457 \pm 2,469 \mu \mathrm{m}$ y a nivel central $9,562 \pm 1,528$ $\mu \mathrm{m}$.

A los 14,5 dpc ocurre la diferenciación del iris. La diferenciación del iris tiene lugar en la copa óptica donde se encuentran las retinas neural y pigmentaria. El iris rodea la parte exterior del lente, contiene una capa epitelial interna (IRI) que mide $25,502 \pm 2,501 \mu \mathrm{m}$ y una capa epitelial externa (IRE) cuyo espesor es de 13,097 $\pm 1,977 \mu \mathrm{m}$, que se continúan con las capas neural que en la periferia mide $65,173 \pm 5,871 \mu \mathrm{m}$ y a nivel central $222,929 \pm 22,213 \mu \mathrm{m}, \mathrm{y}$ pigmentaria de la retina con un espesor periférico de $26,045 \pm 4,231 \mu \mathrm{m}$ y central de $12,286 \pm 1,719 \mu \mathrm{m}$. Se observan 8-10 capas celulares mesenquimales (Fig. 2). 
A los $17 \mathrm{dpc}$, la altura del epitelio de la capa nerviosa de la retina se concentra en la parte central midiendo $160,357 \pm 11,978 \mu \mathrm{m}$ y disminuye hacia la región periférica a $102,111 \pm 5,789 \mu \mathrm{m}$. La capa externa (o pigmentaria) de la retina varia en forma inversa desde la parte media con $7,756 \pm 0,951 \mu \mathrm{m}$ hasta la región mas periférica donde aumen-

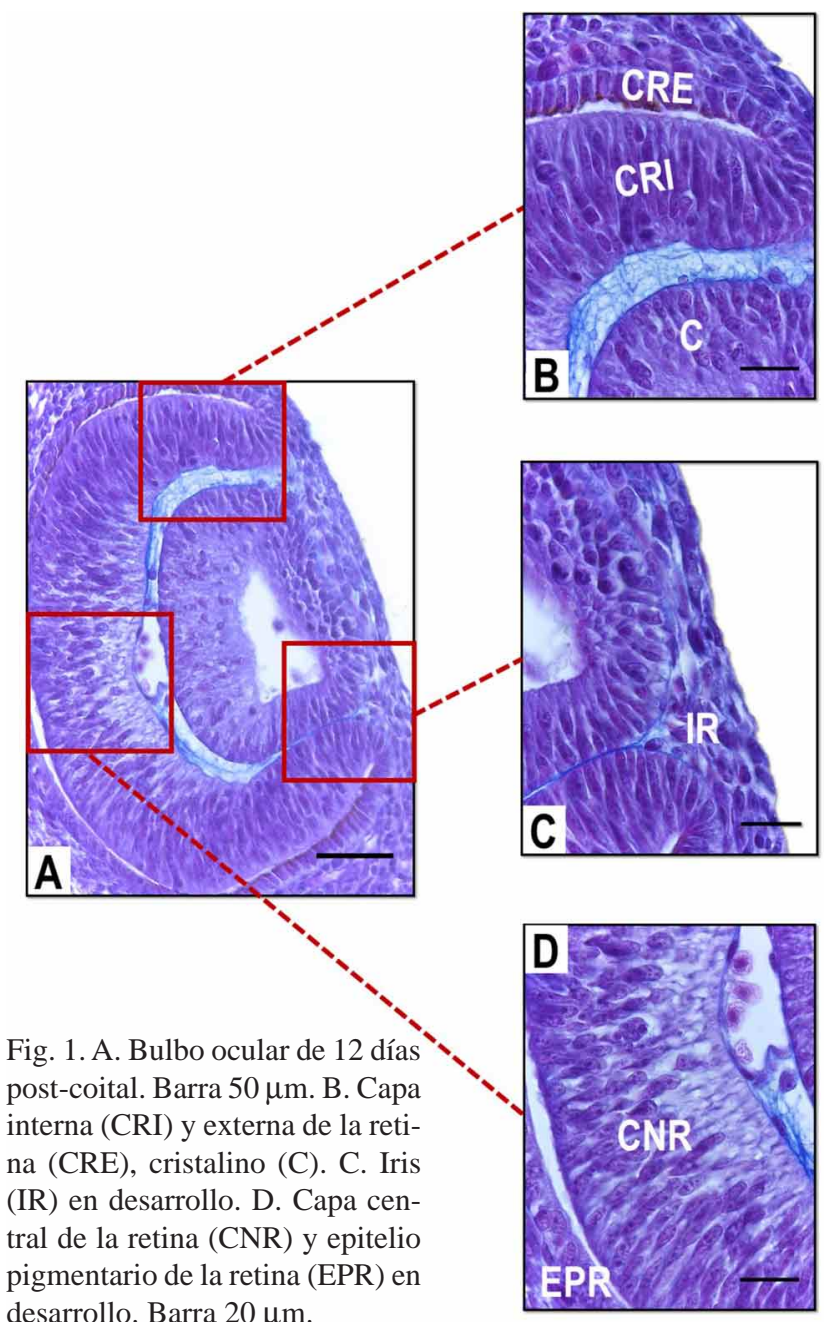

ta a $12,911 \pm 1,539 \mu \mathrm{m}$. Aún no se ha constituido el estroma del iris, tampoco esta formado el cuerpo ciliar. La IRI mide

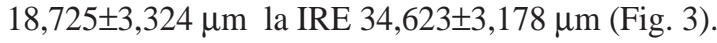

Al comparar las diferentes alturas, hubo diferencias significativas en relación a la capa externa del iris, donde se evidenció un aumento en su longitud entre los 14,5 a 17 dpc. La capa nerviosa de la retina en su porción central y la capa pigmentaria de la retina en su porción periférica, evidenciaron diferencias significativas entre todos los grupos, mostrando un espesor mayor a las 14,5 dpc, seguido por el grupo de $17 \mathrm{dpc}$ y el menor lo presentaron los embriones de 12,5 dpc. En la porción periférica, existió una progresión lineal de menor a mayor según el avance de la gestación, con diferencias significativas (Tabla I).

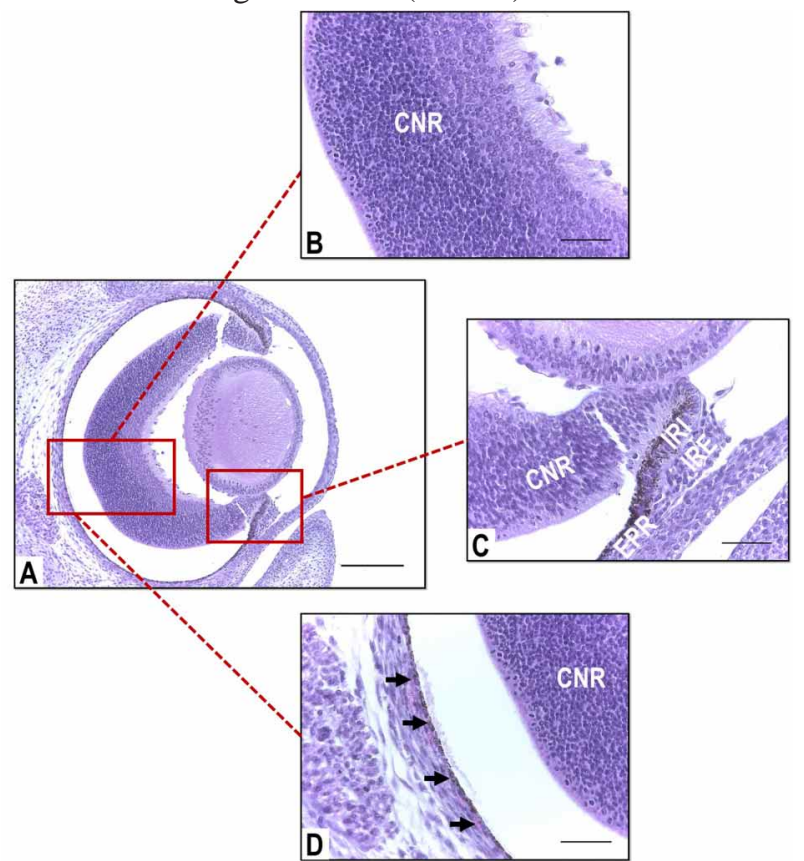

Fig. 2. A. Bulbo ocular de 14,5 días post-coital. Barra $200 \mu \mathrm{m}$. B. Capa neural de la retina (CNR) en su porción central. C. Capa neural de la retina (CNR), epitelio interno del iris (IRI), epitelio externo del iris (IRE), epitelio pigmentario de la retina (EPR) en su porción periférica. D. Flechas indican epitelio pigmentario de la retina.Barra $50 \mu \mathrm{m}$.

Tabla I. Longitud de las capas del iris y retina a los 12, 14,5 y 17 días post-coital.

\begin{tabular}{lccc}
\hline Capa & $12 \mathrm{dpc}$ & $14,5 \mathrm{dpc}$ & $17 \mathrm{dpc}$ \\
\hline Capa interna del iris $(\mu \mathrm{m})$ & - & $25,502 \pm 2,501$ & $18,725 \pm 3,324$ \\
Capa externa del iris $(\mu \mathrm{m})$ & - & $13,097 \pm 1,977 \mathrm{a}$ & $34,623 \pm 3,178 \mathrm{a}$ \\
Capa nerviosa de la retina (central) $(\mu \mathrm{m})$ & $67,326 \pm 3,264 \mathrm{~b}$ & $222,929 \pm 22,213 \mathrm{~b}$ & $160,357 \pm 11,978 \mathrm{~b}$ \\
Capa nerviosa de la retina (periférica) $(\mu \mathrm{m})$ & $36,069 \pm 1,936^{\mathrm{c}}$ & $65,173 \pm 5,87^{\mathrm{c}}$ & $102,111 \pm 5,789 \mathrm{c}$ \\
Capa pigmentaria de la retina (central) $(\mu \mathrm{m})$ & $9,562 \pm 1,528$ & $12,286 \pm 1,719$ & $7,756 \pm 0,951$ \\
Capa pigmentaria de la retina (periférica) $(\mu \mathrm{m})$ & $13,457 \pm 2,469 \mathrm{~d}$ & $26,045 \pm 4,231 \mathrm{~d}^{\mathrm{e}}$ & $12,911 \pm 1,539 \mathrm{e}$ \\
\hline
\end{tabular}

dpc: días post-coital; (a) diferencias entre los 14,5 y $17 \mathrm{dpc}$ con valor $\mathrm{p}<0,001$; (b) y (c) diferencias entre todos los grupos con valor $\mathrm{p}<0,001$; (d) diferencias entre 12 y 14,5 dpc con valor $\mathrm{p}<0,005$ y (e) diferencias entre 14,5 y $17 \mathrm{dpc}$ con valor $\mathrm{p}<0,001$. 
En relación a la inmunotinción, la capa epitelial interna del iris presenta una parte con inmunotinción positiva al anticuerpo anti-SHH y otra parte que constituye un epitelio de células cilíndricas simples negativas a este anticuerpo. La capa epitelial externa presenta epitelio cilíndrico simple negativos al anticuerpo anti-SHH. Las capas de la retina también son positivas, como también la pared externa del lente (Fig. 3).

\section{DISCUSIÓN}

En este estudio se ha reconocido cambios histológicos de los derivados de la copa óptica y del mesénquima periocular. También se observó la presencia de Sonic hedgehog (Shh) en las capas que constituyen el esbozo de iris en los períodos más avanzados del desarrollo, como a las $17 \mathrm{dpc}$.

Sobre los cambios histológicos de los derivados periféricos de la copa óptica, cuando ha transcurrido el 57 $\%$ de la gestación, se evidencia el desarrollo del iris. Es así como a los 12 dpc ocurren cambios significativos tanto en la altura del epitelio de la capa nerviosa de la retina, como también de la capa externa. La capa nerviosa de la retina disminuye en altura desde el centro hacia la periferia, en cambio la capa externa (que en esta cepa de ratón no es pigmentada) aumenta su espesor hacia la periferia, para formar las capas interna y externa del iris. Esto se relaciona con lo observado por Davis-Silberman \& Ashery-Padan quienes indican que la diferenciación neuronal progresa desde la retina central hacia la periferia hasta alcanzar el margen de la copa óptica, la cual contiene los progenitores del iris y cuerpo ciliar. En las etapas siguientes, es decir a los 14,5 dpc y $17 \mathrm{dpc}$, estos cambios se acentúan en el iris, lo cual es similar a lo observado por el mismo grupo de estudio, quienes indican que los progenitores ya se han diferenciado de la neuroretina y no expresan marcadores neuronianos BIII-tubulina, como tampoco hay mitosis. A los $17 \mathrm{dpc}$ no se ha constituido el estroma del iris, ni tampoco el cuerpo ciliar.

Sobre los cambios histológicos del mesénquima periocular, sólo hubo un aumento de las capas de celulares migratorias conforme avanza la gestación. De acuerdo con Reza \& Yasuda (2004) estas se encontraban entre el ángulo de la futura córnea y el margen de la copa óptica.
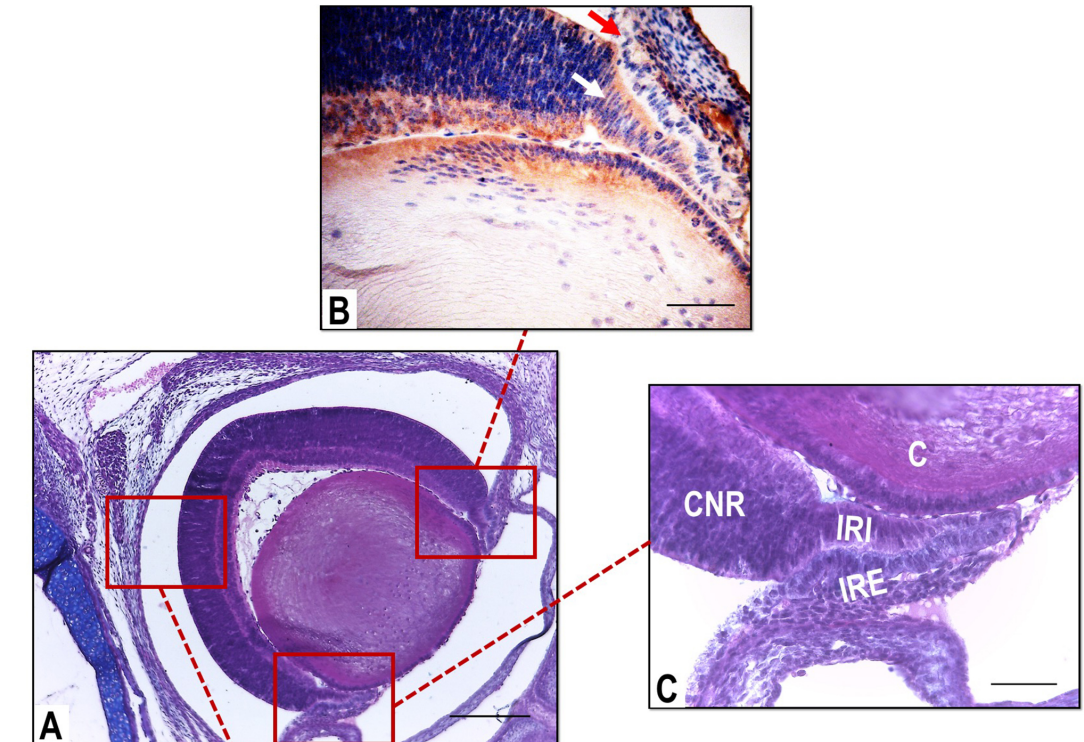

(1)

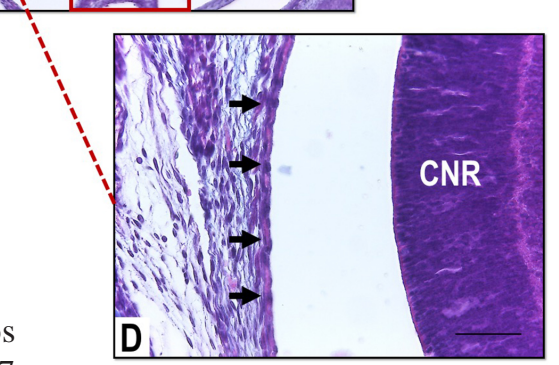

Fig. 3. A. Bulbo ocular a los 17 días post-coital. Barra $200 \mu \mathrm{m}$. B. Inmunotinción con anticuerpo anti-Shh. Flecha blanca indica capa interna del iris inmunopositiva y fecha roja indica capa externa del iris negativa a Shh. C. Capa neural de la retina (CNR), cristalino (C), epitelio interno del iris (IRI), epitelio externo del iris (IRE). D. Capa neural del aretina (CNR), flechas indican epitelio pigmentario de la retina.

En el caso del reconocimiento de la presencia de Sonic hedgehog (Shh) en las capas que constituyen el esbozo de iris, observamos que a los 17,5 dpc existe una inmunotinción positiva del morfógeno Shh en el primer segmento de la capa interna del iris. Este epitelio pierde completamente la inmunotinción en el último segmento del iris. Por el contrario, la capa externa del iris es completamente negativa al anticuerpo anti-SHH. Otras estructuras que resultaron positivas fueron la capa ganglionar de la retina. Esto último concuerda con los estudios realizados por Perron et al. (2003) en los cuales indica que Shh se expresa en la capa ganglionar de la retina. Además ha sido demostrado que Shh juega un rol importante en la diferenciación de fotoreceptores y en el control de poblaciones de células ganglionares (Levine et al., 1997; Zhang \& Yang, 2001). En estudios realizados en alevines de Salmón Salmo salar observamos inmunotinción positiva en las células ganglionares y capa nuclear interna de la retina (Rojas et al., 2016). 
También observamos la inmunotinción positiva del epitelio del lente. Varios autores han enfatizado el rol del lente en el desarrollo de las estructuras del segmento anterior, incluyendo el iris (Beebe, 1986; Davis-Silberman \& Ashery-Padan). Por ejemplo si se retira el lente, se impide el desarrollo del iris, cuerpo ciliar y cornea (Beebe \& Coast, 2000).

En los salmones existe una zona proliferativa germinativa formada por células con morfología fusiforme, que corresponde a un remanente del neuroepitelio embrionario (Pellón et al., 2015). Esto es similar a lo descrito en la retina adulta donde Stenkamp \& Frey (2003) describe que se generan nuevas células a partir de dos poblaciones de retinoblastos, la zona periférica germinal (ZPG) y el linaje de los progenitores de bastones. Desde la ZPG se forman todos los tipos celulares retinianos excepto los bastones (Otteson \& Hitchcock, 2003).

A los 17 dpc aún no está constituido el estroma del iris, su formación es lenta y tardía, incluso continúa en el período postnatal. Esto se relaciona con la característica fenotípica con las cuales nace el ratón, ya que ellos nacen con los ojos cerrados y solo los abren a los 5 días postnatales (Boatright et al., 2015).

\section{CONCLUSIONES}

En el período fetal tardío se observa la inmunotinción positiva de Sonic hedgehog (Shh) en el lente, en el primer segmento de la capa interna del esbozo del iris y en la capa ganglionar de la retina. La expresión de este morfógeno a los $17 \mathrm{dpc}$ se relaciona con la diferenciación tardía del iris y con los ojos cerrados de las crías al nacimiento.

PELLÓN, M.; CONEI, D.; SAINT-PIERRE, G.; SMOK, C.; DONOSO, V.; DEL SOL, M. \& ROJAS, M. Embryo-fetal Development of iris: immunohistochemestry of morphogen Shh. Int. J. Morphol., 37(1):221-226, 2019.

SUMMARY: In order for the iris to develop, a specification of the peripheral layer of the optic cup to a non-neuronal target is required, as well as the migration of periocular mesenchymal cells. Our aim was to recognize the histological changes of peripheral derivatives of the optic cup and periocular mesenchyme, as well as recognize the presence of the morphogen Sonic hedgehog (Shh) in the layers constituting the outline of the iris. 15 female mice (Mus musculus) pregnant young adults were used. Euthanasia was performed with sodium thiopental. Embryos and fetuses of 12, 14.5 and 17 days post-coital (dpc) were processed with histological and immunohistochemical technique with anti-Shh antibody (scbt, H-
160, rabbit) with dilution 1:100 in PBS. At $12 \mathrm{dpc}$, an optic cup showing internal and external retinal layers is observed, and the iris is not observed. Between the lens and the superficial ectoderm, 4 layers of mesenchymal cells are identified. At $14.5 \mathrm{dpc}$, the iris contains two epithelial layers (internal and external) that are continued with the neural and pigmentary layers of the retina. 8 layers of mesenchymal cells are observed. At $17 \mathrm{dpc}$, the inner epithelial layer of the iris presents a more elongated segment with positive immunostaining to Shh and another part that constitutes an epithelium of simple cylindrical cells negative to this antibody. The outer epithelial layer presents the same immunonegative epithelium. The layers of the retina are also positive, as well as the periphery of the lens. The iris is not formed nor is the ciliary body.The immunopositivity in the lens, in the first segment of the inner layer of the iris outline and in the retinal ganglion layer at $17 \mathrm{dpc}$, is related to the late differentiation of the iris and the closed eyes of the offspring at birth.

KEY WORDS: Iris; Eyeball; Sonic hedgehog; Embryonic Development.

\section{REFERENCIAS BIBLIOGRÁFICAS}

Ashery-Padan, R.; Marquardt, T.; Zhou, X. \& Gruss, P. Pax6 activity in the lens primordium is required for lens formation and for correct placement of a single retina in the eye. Genes Dev., 14(21):2701-11, 2000.

Beebe, D. C. \& Coats, J. M. The lens organizes the anterior segment: specification of neural crest cell differentiation in the avian eye. Dev. Biol., 220(2):424-31, 2000.

Beebe, D. C. Development of the ciliary body: a brief review. Trans. Ophthalmol. Soc. U. K., 105(Pt. 2):123-30, 1986.

Boatright, J. H.; Dalal, N.; Chrenek, M. A.; Gardner, C.; Ziesel, A.; Jiang, Y.; Grossniklaus, H. E. \& Nickerson, J. M. Methodologies for analysis of patterning in the mouse RPE sheet. Mol. Vis., 21:40-60, 2015.

Cvekl, A. \& Tamm, E. R. Anterior eye development and ocular mesenchyme: new insights from mouse models and human diseases. Bioessays, 26(4):374-86, 2004.

Davis-Silberman, N. \& Ashery-Padan, R. Iris development in vertebrates; genetic and molecular considerations. Brain Res., 1192:17-28, 2008.

Glaser, T.; Jepeal, L.; Edwards, J. G.; Young, S. R.; Favor, J. \& Maas, R. L. PAX6 gene dosage effect in a family with congenital cataracts, aniridia, anophthalmia and central nervous system defects. Nat. Genet., 7(4):463-71, 1994.

Levine, E. M.; Roelink, H.; Turner, J. \& Reh, T. A. Sonic hedgehog promotes rod photoreceptor differentiation in mammalian retinal cells in vitro. J. Neurosci., 17(16):6277-88, 1997.

Otteson, D. C. \& Hitchcock, P. F. Stem cells in the teleost retina: persistent neurogenesis and injury-induced regeneration. Vision Res., 43(8):92736,2003 .

Pellón, M.; Rojas, M.; Yaikin, P. \& del Sol, M. Morphological study of the retina of salmon (Salmo salar). Int. J. Morphol., 33(2):788-93, 2015.

Perron, M.; Boy, S.; Amato, M. A.; Viczian, A.; Koebernick, K.; Pieler, T. \& Harris, W. A. A novel function for Hedgehog signalling in retinal pigment epithelium differentiation. Development, 130(8):1565-77, 2003.

Reza, H. M. \& Yasuda, K. Lens differentiation and crystallin regulation: a chick model. Int. J. Dev. Biol., 48(8-9):805-17, 2004.

Rimkus, T. K.; Carpenter, R. L.; Qasem, S.; Chan, M. \& Lo, H. W. Targeting the sonic hedgehog signaling pathway: review of smoothened and GLI inhibitors. Cancers (Basel), 8(2):E22, 2016. 
Rojas, M.; Saint-Pierre, G.; Hartley, R.; Vásquez, B.; Conei, D. \& del Sol, M. Immunolocalization of morphogen sonic hedgehog in salmon fry (Salmo salar). Int. J. Morphol., 34(2):770-4, 2016.

Stenkamp, D. L. \& Frey, R. A. Extraretinal and retinal hedgehog signaling sequentially regulate retinal differentiation in zebrafish. Dev. Biol., 258(2):349-63, 2003.

Sukhikh, G. T.; Panova, I. G.; Smirnova, Y. A.; Milyushina, L. A.; Firsova, N. V.; Markitantova, Y. V.; Poltavtseva, R. A. \& Zinov'eva, R. D. Expression of transforming growth factor-b2in vitreous body and adjacent tissues during prenatal development of human eye. Bull. Exp. Biol. Med., 150(1):117-21, 2010.

Tabata, T. \& Takei, Y. Morphogens, their identification and regulation. Development, 131(4):703-12, 2004

Wallace, V. A. Proliferative and cell fate effects of Hedgehog signaling in the vertebrate retina. Brain Res., 1192:61-75, 2008

Zhang, X. M. \& Yang, X. J. Regulation of retinal ganglion cell production by Sonic hedgehog. Development, 128(6):943-57, 2001.
Dirección de correspondencia:

Dra. Mariana Rojas R.

Laboratorio de Embriología Comparada

Programa de Anatomía y Biología del Desarrollo

Facultad de Medicina, ICBM

Universidad de Chile

Santiago - Chile

Email: dramrojas@hotmail.com - mrojasr@u.uchile.cl

Recibido : 10-10-2018

Aceptado: 31-12-2018 